\title{
An Ocellar "Pupil" that Does Not Change with Light Intensity, but with the Insect Age in Triatoma infestans
}

\author{
Teresita C Insausti ${ }^{+}$, Claudio R Lazzari
}

\author{
Departamento de Biología, Facultad de Ciencias Exactas y Naturales, Universidad de Buenos Aires, \\ Ciudad Universitaria (1428), Buenos Aires, Argentina
}

The simple eyes (ocelli) of recently emerged adult Triatoma infestans exhibit a narrow elongated "pupil", surrounded by a ring of brown-reddish pigment, the "iris". This pupil does not respond to changes in the illumination, but varies in size after the imaginal ecdysis. This change corresponds, internally, with the growth of the corneal lens and the associated retina up to an age of about 20 days. This has not been previously observed in an insect. The use of this characteristic for recognising young adults of this species is suggested.

Key words: Triatoma - simple eyes - ocelli - Chagas vectors - ageing

The simple eyes or ocelli of adult insects have been the subject of numerous anatomical, physiological, and behavioural investigations (reviewed by Goodman 1970, 1981, Mizunami 1994, 1995). Different functional roles have been ascribed to these organs, e.g., modulation of muscular tone for the flight system, flight equilibrium control (Wilson 1978, Stange \& Howard 1979, Stange 1981, Rowell \& Pearson 1983), orientation in walking flies (Wehrhahn 1984) and ants (Fent \& Wehner 1985) and, possibly, the dorsal light response used during flight (Kalmus 1945, Goodman 1965).

Triatoma infestans (Klug) (Hemiptera: Reduviidae), a main vector of Chagas disease, are haematophagous bugs highly adapted to domestic environments. Their two ocelli are located behind the compound eyes, looking dorsolaterally and frontally. The ocelli are well developed with prominent spherical lenses overlying the photoreceptor layer (Fig. 1A) (Insausti 1997). Descending firstorder interneurones project to contralateral posterior slope, deutocerebrum and suboesophageal, pro-, meso- and metathoracic centres (Insausti \& Lazzari 1996). Recently, it has been demonstrated, for the first time in an insect, that the ocelli of $T$.

This work received financial support from the Universidad de Buenos Aires, Conicet/Argentina, and the WHO/UNDP/World Bank Special Programme for Research and Training in Tropical Diseases.

+Corresponding author. Fax: +54 11-4576. 3384/4544. 7893. E-mail: lazzari@bg.fcen.uba.ar

Received 20 September 1999

Accepted 22 March 2000 infestans are able to mediate, by their own, the negative phototactic behaviour of the bugs, i.e., but not through the modulation of the sensitivity of the compound eyes (Lazzari et al. 1998).

Screening pigment granules can be found in different locations within the ocelli of $T$. infestans inside several layers of pigment cells. These oval cells are filled by granules of brown-reddish pigment, giving place to an "iris", that limits an ovoid "pupil", whose major axis runs horizontally (Insausti 1997). The extent of this pupil was observed to differ among individuals. Pigmentary irises have been described for the ocelli of other insects (Wilson 1975, Stavenga et al. 1979) and also in the compound eyes of the related species Rhodnius prolixus (Müller 1970). In all cases, such an iris exhibits dynamic changes associated to light intensity. The present work analyses the changes occurring in the pupil of T. infestans, as well as its morphological basis and dynamics.

\section{MATERIALS AND METHODS}

Adults of Triatoma infestans of both sexes were reared in the laboratory at $28^{\circ} \mathrm{C}$ and fed on citrated sheep blood with the aid of an artificial feeder (Núñez \& Lazzari 1990).

To test the changes in the ocellar pupil related to light intensity, groups of insects exhibiting differences in the pupil size were exposed during $24 \mathrm{~h}$ either to bright illumination provided by a tungsten lamp (4000 lux) or to total darkness (0 lux).

Pupil size was further measured and related to the bug's age. For this, males and females of $T$. infestans were individually marked with non-toxic acrylic paint and the dimensions of the ocellar pupil were measured with the aid of a stereomicroscope and photographed at different times after the imaginal ecdysis. 
The morphological basis of the variation in the pupil size was analyzed. Light microscopy was performed on bugs' heads fixed in a mixture of $2.5 \%$ glutaraldehyde and $2 \%$ paraformaldehyde in phosphate buffer $(\mathrm{pH} 7.3)$ and postfixed with $1 \%$ osmium tetroxide. After dehydration, they were embedded in Durcupan, cut in sections $5 \mu \mathrm{m}$ thick and stained with methylene blue.

\section{RESULTS}

No external changes in the distribution of the pigments that form the ocellar iris could be observed in relation to illumination. However, a marked variation with the insect age was found in the pigment distribution of the ocelli (Figs 1B, C, D, 2). In young adults, the pupil looks like a narrow horizontal strip of about $250 \mu \mathrm{m}$ length and $37 \mu \mathrm{m}$ wide, with the rest of the cornea internally covered by pigments (Fig. 1B). After approximately three weeks, the ocellar iris is reduced to a narrow peripheral ring, and the pupil reaches $300 \mu \mathrm{m}$ length and $200 \mu \mathrm{m}$ wide (Figs 1C, D). The pupil remains in this stage, along the rest of the bugs' life, without noticeable changes.

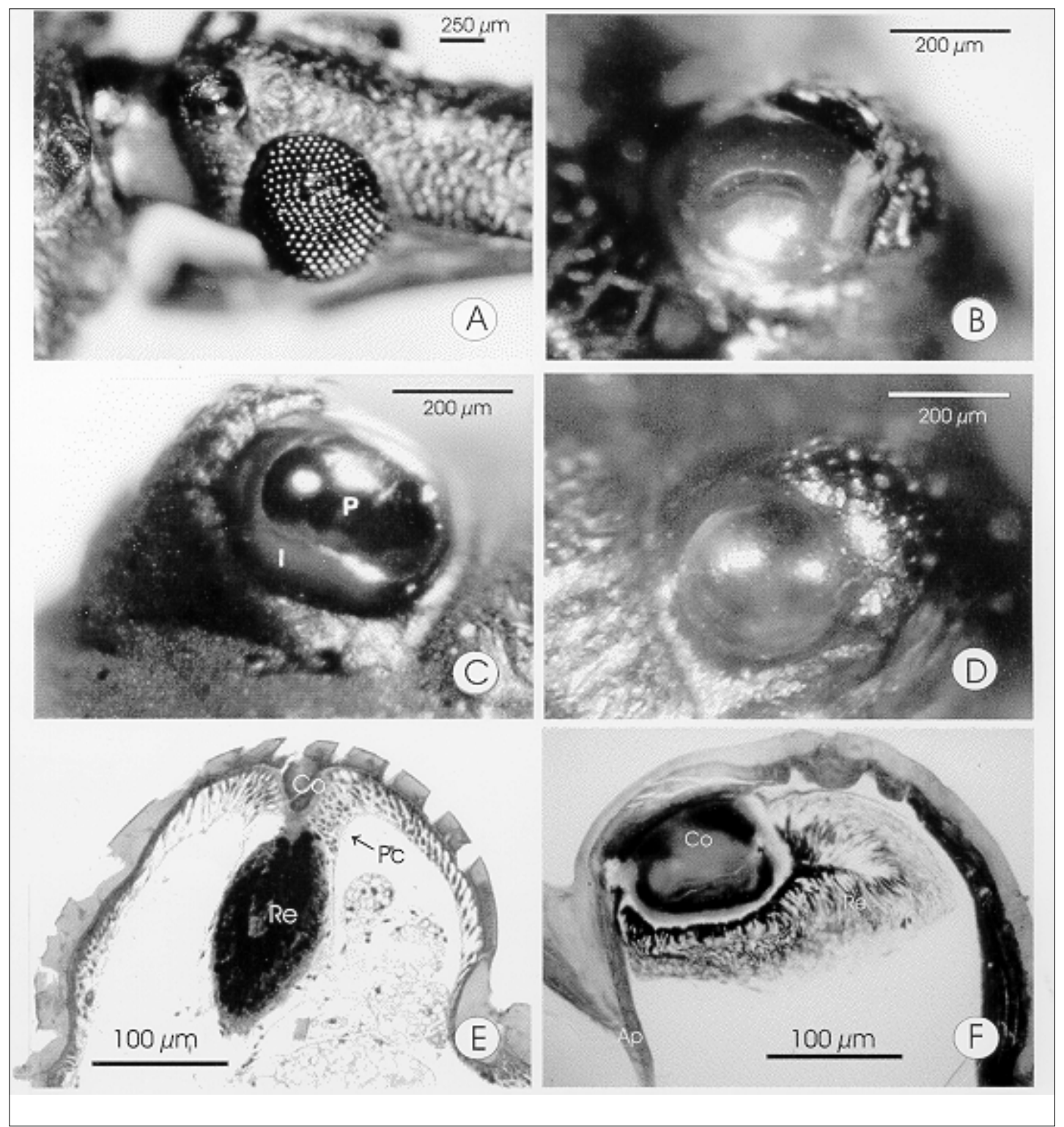

Fig. 1 - A: lateral view of the head of Triatoma infestans, seen with the aid of the stereomicroscope. The right ocellus and compound eye are shown; B: lateral view of the ocellus of a recently emerged adult; C: ocellus of one-week-old adult; D: ocellus of more than 20 days; E: section through the ocellus of a recently emerged adult; F: section through the ocellus of a 20 days old insect. Ap: apodeme; Co: cornea; I: iris; P: pupil; Pc: pigmentary cells; Re: retinular layer 


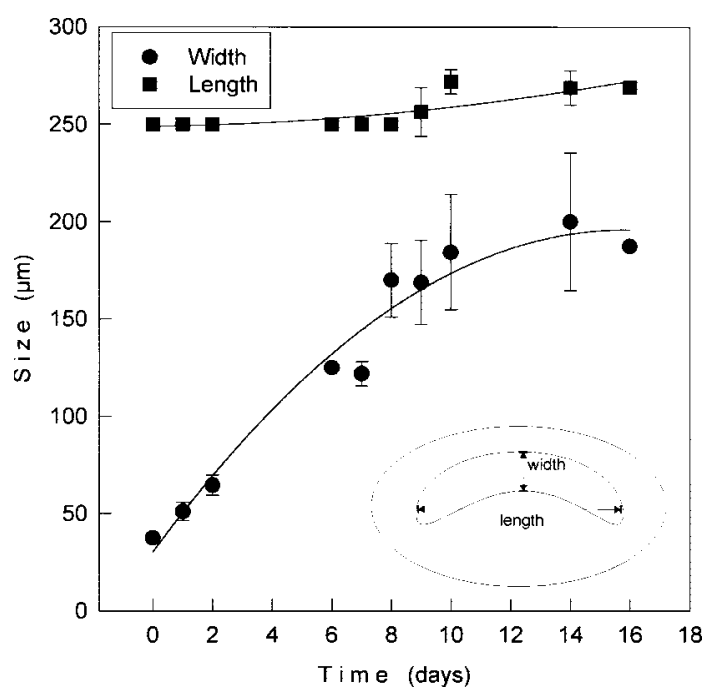

Fig. 2: dimensions of the ocellar pupil of Triatoma infestans as a function of the time after the imaginal ecdysis. Mean \pm s.e.m. from measurements performed in ten insects (five males and 5 females) are depicted.

Histological methods revealed that changes in the size of the ocellar pupil of $T$. infestans with age correspond to changes in the cornea and in the underlying retina (Figs 1E, F). Fig. 1E shows a section through the ocellus of a bug recently emerged. The ocelli are not yet fully developed and the cornea and retina continue to grow during the first weeks of the adult life. The pupil corresponds internally to the portion of thickened cornea, with the retina restricted to this zone. The iris corresponds to a ring-like layer of pigmentary cells that becomes narrower with the insect age. Fig. $1 \mathrm{~F}$ shows the fully developed ocellus of a 20 days old insect, in which the cornea and the retina are fully developed.

\section{DISCUSSION}

Pigmentary irises have been described in compound (Müller 1970) and simple eyes (Wilson 1975, Stavenga et al. 1979), as structures controlling photon influx into the photoreceptor cells. In the case of T. infestans, however, the ocellar pupil does not perform dynamic changes associated to incident light, but varies with the insect age. To our knowledge, this is the first report of such a phenomenon in an insect.

In T. infestans, what we observe as an iris is a layer of pigmentary epithelium that lies beneath the thin undeveloped cornea. The pupil corresponds to the thickened areas of the cornea that grow from a narrow curved band to occupy the whole cavity of the ocellus. It could be hypothesised that the dynamic changes in the pigments are more related with the late development of the ocelli, taking place after the eclosion to adult. However, other hemimetabolous insects, such as locusts, present ocelli that reach a high degree of differentiation already during the embryonic life (Mobbs 1979), and almost complete their development during the larval growth. This pattern appears notably different from that reported here for T. infestans. Firstly, the larvae of this bug lacks ocellar lenses. Secondly, the growth of the cornea and the associated retina during the early adult life starts from a thin lens with a narrow band of thickened cuticle, that expands dorso-ventrally to become an almost spherical thick ocellar lens (Insausti 1997).

Although a functional role of the ocelli has been revealed in relation to phototactic behaviour in $T$. infestans (Lazzari et al. 1998), the available information on the biology of triatomine bugs does not allow us to exclude a possible function in flight control. According to the function of the ocelli in other insects, the pupil growth can be correlated with the flight activity of $T$. infestans. As shown by Lehane and Schofield (1982), the maximum flight activity occurs on the 20th day of imaginal life of males and females. This time period is in agreement with the time when the ocelli complete their development.

A remarkable aspect is that the ocellar pupil of T. infestans constitutes a potential indicator of the age of this insect and therefore could provide a useful tool for epidemiological studies. The pupil can be easily seen in the field with the aid of a magnifying glass and can be measured in the laboratory under a stereomicroscope. Although this indicator is limited in the time interval relative to the extension of the imaginal life, it is especially useful during a life period that exhibits a great ecological and epidemiological relevance. Dispersive flights and reproductive activity are two highly relevant activities, from an epidemiological point of view, that initiate or increase at about 20 days after the imaginal ecdysis. The development of the ocellar pupil could be an indicator to help us to discern whether a given adult insect found in the field has emerged in the same pace, or could have arrived there flying. Therefore, developing curves of ocellar pupiles for several triatomine species under different environmental conditions are being studied.

\section{ACKNOWLEDGMENTS}

To M Giurfa for correcting the manuscript and to the staff members of their laboratory for many fruitful discussions. 


\section{REFERENCES}

Fent K, Wehner R 1985. Ocelli: a celestial compass in the desert ant Cataglyphis. Science 228: 192-194.

Goodman LJ 1965. The role of certain optomotor reactions in regulating stability in the rolling plane during flight in desert locust Schistocerca gregaria. J Exp Biol 42: 385-407.

Goodman LJ 1970. The structure and function of the insect dorsal ocellus. Adv Insect Physiol 7: 97-195.

Goodman LJ 1981. Organization and physiology of the insect dorsal ocellar system. In H Autrum, Handbook of Sensory Physiology, Vol. VII/6C, SpringerVerlag, Berlin, Heidelberg and New York, p. 201286.

Insausti TC 1997. Estudio del Sistema Ocelar de la Vinchuca Triatoma infestans (Klug, 1834) (Heteroptera: Reduviidae), PhD Thesis, University of Buenos Aires, Argentina, $257 \mathrm{pp}$.

Insausti TC, Lazzari CR 1996. Central projections of first-order interneurons in the bug Triatoma infestans (Heteroptera: Reduviidae). J Morphol 229: 161169.

Kalmus H 1945. Correlations between flight and vision, and particularly between wings and ocelli, in insects. Proc R Entomol Soc London A20: 84-96.

Lazzari CR, Reisenman CE, Insausti TC 1998. The role of the ocelli in the phototactic response of Triatoma infestans (Heteroptera: Reduviidae). J Insect Physiol 44: 1159-1162.

Lehane MJ, Schofield CJ 1982. Flight initiation in Triatoma infestans (Klug) (Hemiptera: Reduviidae). Bull Entomol Res 72: 497-510.

Mizunami M 1994. Information processing in the insect ocellar system: comparative approaches to the evolution of visual processing and neural circuits. Adv Insect Physiol 25: 151-265.

Mizunami M 1995. Functional diversity of neural organization in insect ocellar systems. Vis Res 4: 443452.

Mobbs PG 1979. Development of the dorsal ocelli of the desert locust Schistocerca gregaria Forsk (Orthoptera: Acrididae). Int J Insect Morphol Embryol 8: 237-255.

Müller J 1970. Feinanbau und Dunkelanpassung der Komplexaugen von Rhodnius prolixus. Zool Jahrb Allg Zool Physiol 75: 11-133.

Núñez JA, Lazzari CR 1990. Rearing of Triatoma infestans Klug (Het., Reduviidae) in the absence of a live host. I. Some factors affecting the artificial feeding. J Appl Entomol 109: 87-92.

Rowell CHF, Pearson KG 1983. Ocellar input to the flight motor system of the locust: structure and function. J Exp Biol 103: 265-288.

Stange G 1981. The ocellar component of flight equilibrium control in dragonflies. J Comp Physiol 141: 335-347.

Stange G, Howard J 1979. An ocellar light response in a dragonfly. J Exp Biol 83: 351-355.

Stavenga DG, Bernard GD, Chappel RL, Wilson M 1979. Insect pupil mechanisms. III. On the pigment migration in dragonfly ocelli. J Comp Physiol 129: 199205.

Wehrhahn C 1984. Ocellar vision and orientation in flies. Proc R Soc London B 222: 409-411.

Wilson M 1975. Autonomous pigment movement in the radial pupil of locust ocelli. Nature 258: 603604.

Wilson M 1978. The functional organisation of locust ocelli. J Comp Physiol 124: 297-316. 